\title{
Stroke and dementia risk: A systematic review and meta-analysis
}

\author{
Elżbieta Kuźma $^{\mathrm{a}, 1}$, Ilianna Lourida ${ }^{\mathrm{a}, 1}$, Sarah F. Moore ${ }^{\mathrm{a}}$, Deborah A. Levine ${ }^{\mathrm{b}, \mathrm{c}, \mathrm{d}}$, \\ Obioha C. Ukoumunne ${ }^{\mathrm{e}}$, David J. Llewellyn ${ }^{\mathrm{a}}{ }^{*}$ \\ ${ }^{a}$ University of Exeter Medical School, St Luke's Campus, Exeter, UK \\ ${ }^{b}$ Department of Internal Medicine, University of Michigan Medical School, Ann Arbor, MI, USA \\ ${ }^{c}$ Institute for Healthcare Policy and Innovation, University of Michigan, Ann Arbor, MI, USA \\ ${ }^{d}$ Department of Neurology and Stroke Program, University of Michigan, Ann Arbor, MI, USA \\ ${ }^{e}$ NIHR CLAHRC South West Peninsula (PenCLAHRC), University of Exeter Medical School, St Luke's Campus, Exeter, UK
}

\begin{abstract}
Introduction: Stroke is an established risk factor for all-cause dementia, though meta-analyses are needed to quantify this risk.

Methods: We searched Medline, PsycINFO, and Embase for studies assessing prevalent or incident stroke versus a no-stroke comparison group and the risk of all-cause dementia. Random effects meta-analysis was used to pool adjusted estimates across studies, and meta-regression was used to investigate potential effect modifiers.

Results: We identified 36 studies of prevalent stroke (1.9 million participants) and 12 studies of incident stroke (1.3 million participants). For prevalent stroke, the pooled hazard ratio for all-cause dementia was 1.69 (95\% confidence interval: $1.49-1.92 ; P<.00001 ; \mathrm{I}^{2}=87 \%$ ). For incident stroke, the pooled risk ratio was 2.18 (95\% confidence interval: $1.90-2.50 ; P<.00001$; $\mathrm{I}^{2}=88 \%$ ). Study characteristics did not modify these associations, with the exception of sex which explained $50.2 \%$ of between-study heterogeneity for prevalent stroke.
\end{abstract}

Discussion: Stroke is a strong, independent, and potentially modifiable risk factor for all-cause dementia.

(C) 2018 the Alzheimer's Association. Published by Elsevier Inc. All rights reserved.

\section{Introduction}

Stroke is associated with the risk of cognitive impairment and dementia [1-3]. A systematic review [3] of 16 studies conducted in 2008 concluded that both history of and new stroke were associated with risk of developing all-cause dementia, although they were not able to conduct a metaanalysis at the time due to methodological heterogeneity in the included studies. A meta-analysis [4] of 30 studies conducted in 2009 established that dementia prevalence in symptomatic stroke patients increased from $10 \%$ before first

Conflicts of interest: The authors declare no conflicts of interest to disclose.

${ }^{1}$ These authors contributed equally to the manuscript

*Corresponding author. Tel.: 0044(0)1392 72 6018; Fax: 0044(0) 1392722894.

E-mail address: david.1lewellyn@exeter.ac.uk stroke to $20 \%$ soon after first stroke, and more than a third had dementia after recurrent stroke. More recently, a metaanalysis [5] of six studies conducted in 2013 established that stroke is a moderately strong risk factor for Alzheimer's disease $(\mathrm{AD})$ (risk ratio $[\mathrm{RR}]=1.59,95 \% \mathrm{CI}=1.25-2.02$ ). Taken together, these studies highlight the central causal role of symptomatic stroke, rather than the underlying vascular risk factors. Given the current lack of disease-modifying treatments and the complexity of multiple pathologies contributing to dementia, estimating the excess risk of dementia after stroke has the potential to inform preventive strategies to reduce the global burden of dementia. A recent umbrella review identified that no previous meta-analysis of the relationship between stroke and all-cause dementia had been undertaken [6]. A large number of original studies have been published since the systematic review conducted in 2008 [3]. Our objective was therefore to conduct the first 
meta-analysis of the relationship between stroke and allcause dementia risk.

\section{Methods}

We updated the systematic review conducted by Savva et al. [3] and performed study-level random effects metaanalyses after general guidance provided by the Center for Reviews and Dissemination, UK [7].

\subsection{Search strategy and selection criteria}

Following the methods of the previous systematic review [3] and our predefined protocol, we developed search strategies for Medline, PsycINFO, and Embase (via OvidSP), including subject headings and free text terms relevant to dementia, stroke, and study design (Supplementary Appendix A, Methods, and Fig. A1, A2, A3). We conducted our searches on April 27, 2017 (E.K.), restricting them to studies published after 2008 to avoid overlap with the previous systematic review which searched up to December 31, 2008 [3]. We also conducted backward and forward citation searches (via Web of Science; by the authors E.K. and I.L.) of publications included through our searches and in the previous systematic review [3]. We included prospective studies published in English investigating the association between prevalent or incident stroke and incident all-cause dementia. The population was adults aged 18 years or older, and the comparison group was adults without prevalent or incident stroke. Prevalent stroke was defined as history of previous stroke at baseline and incident stroke as stroke occurrence during follow-up. Studies with outcomes other than allcause dementia, that is, dementia subtypes or dementiarelated outcomes (e.g., neuroimaging or biomarkers) were excluded. We also excluded studies with no comparison group or comparison group other than no stroke (i.e., stroke subtype), animal studies, case reports, narrative reviews, letters, editorials, opinions, book chapters, conference abstracts, and duplicate publications using the same data. Following the predefined inclusion and exclusion criteria, two reviewers (E.K. and I.L.) independently screened titles, abstracts, and full texts. Discrepancies were resolved by discussion with a third reviewer (D.J.L.).

Key data were extracted by one reviewer (E.K.) and checked by the second (I.L. or S.F.M.). We also contacted corresponding authors of 18 studies for clarification or if relevant data were not fully reported and received additional data or clarification for 13 studies (see Supplementary Appendix A, Methods for details). Two reviewers (E.K. and I.L.) independently assessed the risk of bias of included studies using the Quality Assessment Tool for Quantitative Studies [8] with discrepancies resolved by discussion. For each included study, components of the tool (selection bias, study design, confounders, blinding, data-collection methods, and withdrawals and drop-outs), and overall risk of bias were rated as "strong," "moderate," or "weak".

\subsection{Data analysis}

Studies were categorized by exposure into those investigating either prevalent or incident stroke. Total number of participants and stroke events were reported based on analytic sample size unless otherwise specified. We conducted random effects meta-analyses using the generic inversevariance method [9] in recognition of the inherent methodological heterogeneity across studies. We used the Review Manager 5.3 software [10] to pool compatible estimates for the associations between prevalent or incident stroke and incident all-cause dementia. We prioritized fully adjusted estimates of effect and extracted unadjusted results only if adjusted models were not available. When a group of studies entered in meta-analysis reported results as hazard ratios (HRs) and RRs, we presented the pooled estimate as a RR [11]. In separate meta-analyses, we combined results from studies reporting odds ratios (ORs). Adjusted estimates of effect were used for our primary analyses. In secondary analyses, we used summary estimates from unadjusted results. In sensitivity analyses, we excluded studies whose samples were limited to participants with prevalent mild cognitive impairment (MCI) or diabetes at baseline or with combined prevalent or incident stroke with transient ischemic attack (TIA). Where results were provided separately on the basis of apolipoprotein $\mathrm{E}$ (APOE) genotype (one or more $\varepsilon 4$ allele versus none) or sex (male/female), we also present these additional stratified results. We investigated heterogeneity using Cochran's Chi-squared test and the I-squared statistic [12]. Funnel plots were obtained to evaluate the presence of publication bias. Where estimates from three or more studies were pooled, we reported $95 \%$ prediction intervals (PIs), which indicate the $95 \%$ range of true HRs (RRs or ORs) across settings that are similar to those in the pooled studies [13]. Studies that could not be included in meta-analyses due to important differences in the outcome (e.g., early onset vs. late-onset dementia) or statistical methods used were synthesized narratively.

We used meta-regression to investigate the effects of previously identified potential moderators of the relationship between stroke and dementia [5]. For prevalent stroke, we fitted meta-regression models by regressing the pooled HR of dementia risk on the following: (1) study setting (community vs. noncommunity); (2) inclusion of TIA in stroke assessment/diagnosis (yes/no); (3) dementia diagnostic criteria used (Diagnostic and Statistical Manual of Mental Disorders/International Classification of Diseases, other); (4) stroke assessment based on self-report only (yes/no); (5) adjustment for at least one vascular risk factor (yes/ no); (6) mean/median age of participants in years; (7) proportion of male participants (\%); (8) year at baseline examination; (9) length of follow-up in years; and (10) study quality (strong vs. moderate/weak). For incident stroke, we fitted meta-regression models by regressing the pooled RR of dementia risk on inclusion of TIA in stroke assessment/ diagnosis, mean/median age of participants in years, 
proportion of male participants (\%), year at baseline examination, length of follow-up in years, and study quality (strong vs. moderate/weak) (there were an inadequate number of studies to investigate the other potential moderators). Meta-regression analyses were performed using the "metareg" command in Stata software, version 14.2 (StataCorp, College Station, TX).

\section{Results}

Database searches resulted in 11,129 records. After removing duplicates, we screened 6893 titles and abstracts and identified 99 for full-text review. Twenty six studies met our eligibility criteria. We also included 16 out of the 17 studies from the previous systematic review [3] and four studies identified via backward and forward citation searches (Fig. 1). We excluded the study by Reitz et al. using data from the Rotterdam Study [14] because of overlap with a more recent publication from the same cohort [15], which had longer follow-up and a larger sample size.

The characteristics of the 46 included studies are shown in Table 1 and Supplementary Appendix B, Tables B1 and B2. Nineteen studies were based in America, 16 in Europe, six in Asia, four in Australia, and one was multinational. Thirty six studies included dementiafree participants at baseline, five studies reported they included cognitively normal population samples, and

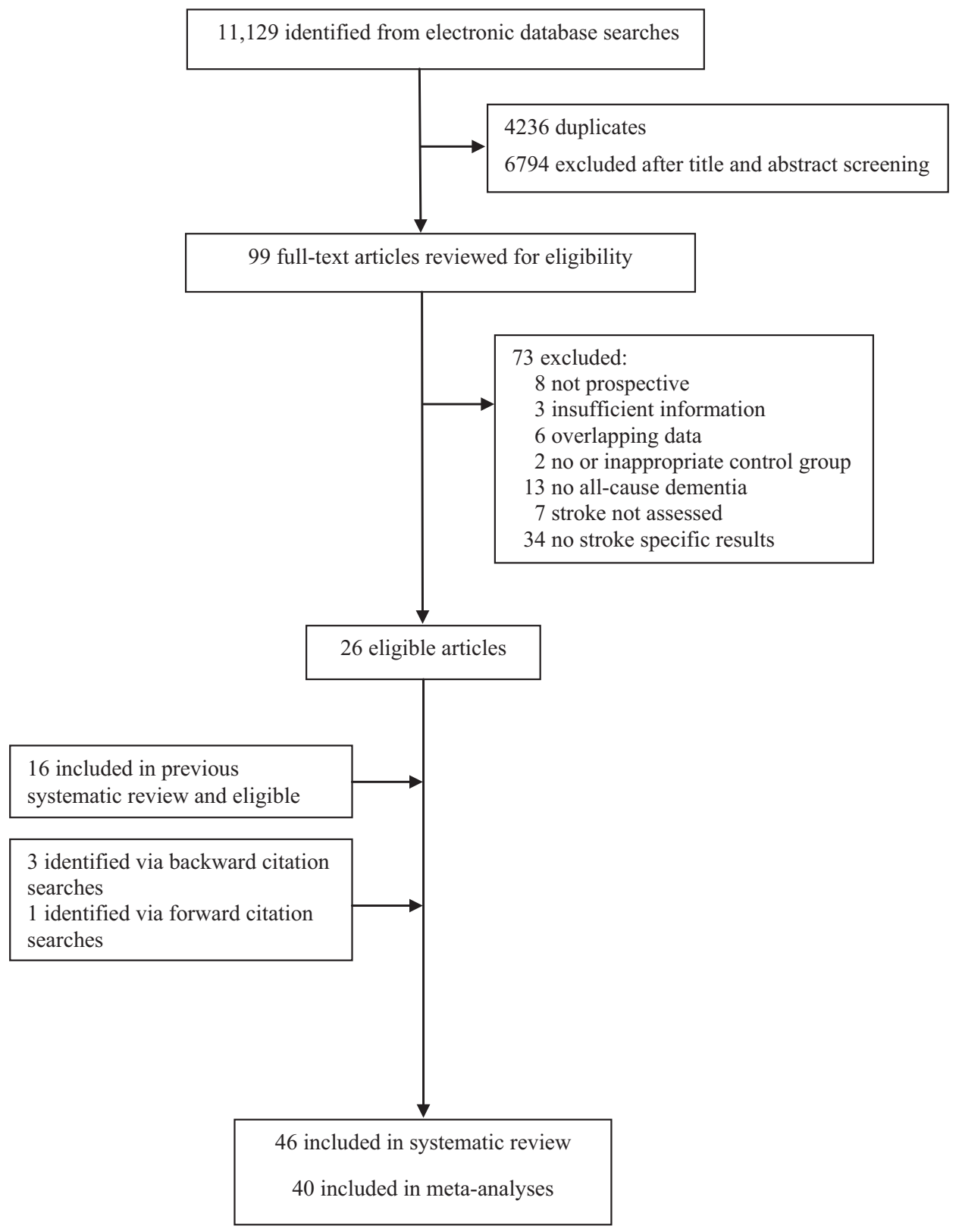

Fig. 1. Flowchart of search results and study retrieval. 
Table 1

Summary of data included in the systematic review*

\begin{tabular}{llll}
\hline & Studies, $^{\dagger}$ & Participants, N & Stroke events, N \\
\hline All studies & 46 & $3,242,618$ & 371,688 \\
$\quad$ Prevalent stroke & 36 & $1,903,733$ & 240,471 \\
$\quad$ Incident stroke & 12 & $1,338,885$ & 131,217 \\
Settings & & & \\
Community & 36 & $1,332,276$ & 225,588 \\
Primary care & 2 & 930,771 & 59,241 \\
Secondary care & 3 & 422 & 64 \\
Other $^{\ddagger}$ & 5 & 979,149 & 86,795 \\
\hline
\end{tabular}

NOTE. Number of participants is based on analytic sample size, and number of stroke events was estimated based on available information, if not clearly reported in the original study.

*Details of individual studies are shown in Supplementary Appendix B, Tables B1 to B4.

${ }^{\dagger}$ Two studies reported on both prevalent and incident stroke exposures.

${ }^{\ddagger}$ Two studies included participants from both primary and secondary care populations, two additional studies included participants from both secondary and community populations, and one study included participants from a military register.

five studies recruited participants with MCI or other cognitive impairment at baseline. Reporting of follow-up varied between studies (e.g., median, mean, or maximum follow-up), and length ranged from nine months to 25 years. Twenty-four studies assessed stroke through self-report or informant report, and 15 studies reported adjudicated dementia diagnosis using Diagnostic and Statistical Manual of Mental Disorders or International Classification of Diseases criteria [16-18]. Five studies assessed both stroke and dementia solely through medical records (Supplementary Appendix B, Tables B3 and B4).

\subsection{Risk of bias}

Sixteen studies were rated as of overall strong quality, 20 as moderate, and ten as weak (Supplementary Appendix B, Table B5). Of the moderate-quality studies, six showed potential bias in the relevant confounders controlled for in the design or analysis, five showed potential bias in datacollection methods, and a further five studies were subject to selection bias. The weak-quality studies showed high risk of bias primarily due to a combination of selection bias $(\mathrm{n}=4)$, data-collection methods $(\mathrm{n}=5)$, confounders $(\mathrm{n}=8)$, and attrition bias $(\mathrm{n}=3)$.

\subsection{Prevalent stroke}

Thirty four prospective cohort studies [19-52] (including three cohort studies of patients with MCI $[19,24,28]$ and one diabetic cohort [22]) and two observational analyses of cohorts recruited for randomized controlled trials [53,54] investigated the association between prevalent stroke and incident all-cause dementia (around 1.9 million participants and 240,471 stroke events; Supplementary Appendix B,
Table B1). Most studies included older adults with an analytic sample size ranging from 52 [28] to 486,640 [25]. Two studies $[26,50]$ included only women.

Pooled results from 22 cohorts of dementia-free participants at baseline $(1,885,536$ participants and 237,886 stroke events) indicated a higher adjusted risk of incident dementia in participants with prevalent stroke compared with those without stroke (pooled HR $=1.69,95 \%$ CI: 1.49-1.92, $P<.00001, \mathrm{I}^{2}=87 \%, 95 \%$ PI: $1.17-2.21$; Fig. 2). Visual inspection of the funnel plot indicated no sign of publication bias (Supplementary Appendix B, Fig. B4). In a sensitivity analysis, we excluded results provided by Walters et al. [49] for those aged 80 to 95 years due to correlation with results reported from the same cohort for those aged 60 to 79 years. The pooled HR remained almost unchanged (1.75, 95\% CI: $1.55-1.97, P<.00001, \mathrm{I}^{2}=78 \%, 95 \%$ PI: 1.33 2.17). In further sensitivity analyses, we excluded studies including participants with MCI $[19,24,32,40]$ or combining stroke with TIA $[24,30,44,48,49,54]$. In both cases, pooled estimates remained essentially unchanged (pooled HR $=1.71,95 \%$ CI: $1.49-1.95, P<.001$, $\mathrm{I}^{2}=89 \%, 95 \%$ PI: $1.17-2.25 ;$ and pooled HR $=1.69$, 95\% CI: $1.46-1.96, P<.001, \mathrm{I}^{2}=51 \%, 95 \%$ PI: $1.23-$ 2.15, respectively; Supplementary Appendix B, Fig. B5.1, B5.2). Meta-regression analyses showed little evidence of effect modification on the basis of study setting $(P=.82)$, inclusion of TIA in stroke assessment/diagnosis $(P=.89)$, dementia diagnostic criteria used $(P=.37)$, stroke assessment based on self-report only $(P=.59)$, adjustment for at least one vascular risk factor $(P=.92)$, mean/median age of participants $(P=.48)$, year at baseline examination $(P=.47)$, length of follow-up $(P=.73)$, or study quality $(P=.75)$. There was however some evidence for effect modification by sex, indicating that the risk of dementia corresponding to prevalent stroke was higher in men than in women $(P=.04)$. Effect modification by sex explained around half of the observed between-study heterogeneity (males: HR $=1.02,95 \%$ CI: $1.00-1.03, P=.04$; females: $\mathrm{HR}=0.98,95 \%$ CI: $0.97-0.99, P=.04 ;$ adjusted $\mathrm{R}^{2}=50.2 \%$ ).

Eight studies [21-23,33,35,46,51,52] reported adjusted ORs instead of HRs (11,336 participants and 1001 stroke events). The pooled estimate indicated increased odds of incident dementia in those with prevalent stroke compared with no prevalent stroke (pooled OR $=1.53,95 \% \mathrm{CI}$ : $1.30-1.80, P<.00001, \mathrm{I}^{2}=0 \%, 95 \%$ PI: $1.22-1.84$; Fig. 3). In a sensitivity analysis, we excluded the study by Bruce et al. [22] as it included only participants with diabetes. The estimate remained essentially unchanged (pooled $\mathrm{OR}=1.57,95 \%$ CI: $1.29-1.91, P<.001, \mathrm{I}^{2}=11 \%, 95 \%$ PI: 1.09-2.05).

In a secondary analysis, the pooled estimate for three studies [26,28,42] reporting unadjusted results (2795 participants and 262 stroke events) indicated little evidence of an association between prevalent stroke and 


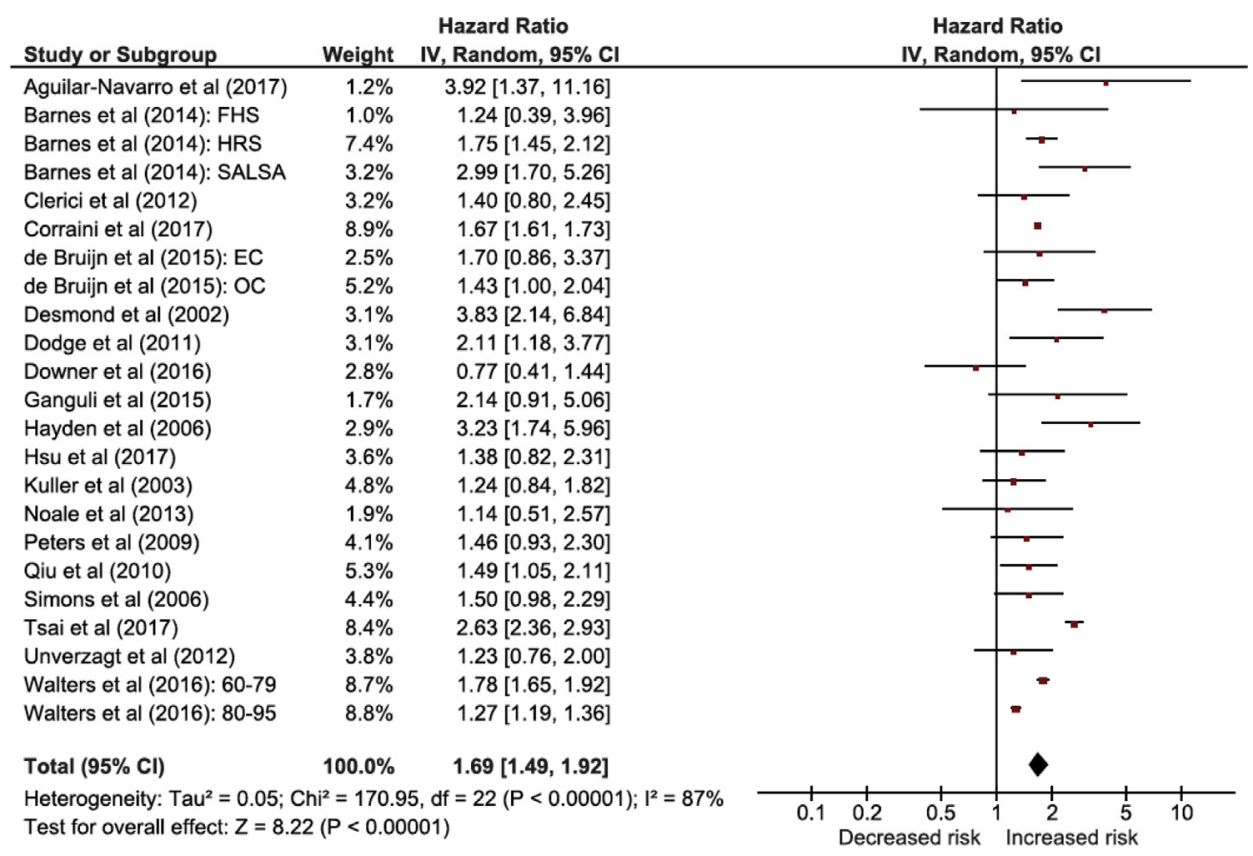

Fig. 2. Meta-analysis of hazard ratios of prevalent stroke compared with no prevalent stroke on incident all-cause dementia. Data are presented as hazard ratios with corresponding weight for each study in the meta-analysis because number of stroke events, dementia cases, and total number of participants were not always available in original included studies. Hazard ratio estimate for the study by Hayden et al. [34] was obtained in Review Manager using the generic inversevariance method and is different from that obtained from a discrete-time survival model reported in the original study (i.e., $\mathrm{HR}=3.23$, CI $=1.74-5.64$ ). The Supplementary Appendix shows the corresponding funnel plot. Abbreviations: IV, inverse-variance estimation method; CI, confidence interval; EC, extended cohort; FHS, Framingham Heart Study; HRS, Health and Retirement Study; OC, original cohort; SALSA, Sacramento Area Latino Study on Aging.

incident dementia (pooled RR $=1.22,95 \%$ CI: 0.50-2.99, $P=.66, \mathrm{I}^{2}=74 \%, 95 \%$ PI: -10.38 to 12.82 ; Supplementary Appendix B, Fig. B5.3). One additional study [47] reported dementia risk according to occurrence of recurrent stroke; both prevalent and recurrent stroke contributed to increased risk of incident dementia compared with absence of stroke (Supplementary Appendix B, Table B3).

Three additional studies $[39,41,50]$ could not be included in the meta-analyses as they did not fully report their results
$[41,50]$ or used standardized morbidity ratio as an effect size which could not be combined with existing estimates [39]. These studies all indicated prevalent stroke was associated with greater risk of incident dementia. We also excluded the study by Hobson et al. [36] from the meta-analysis because it was unclear whether it included participants with prevalent dementia at baseline. The authors reported that controlling for baseline dementia, prevalent stroke more than doubled the risk of incident dementia although there was a high degree of uncertainty surrounding their

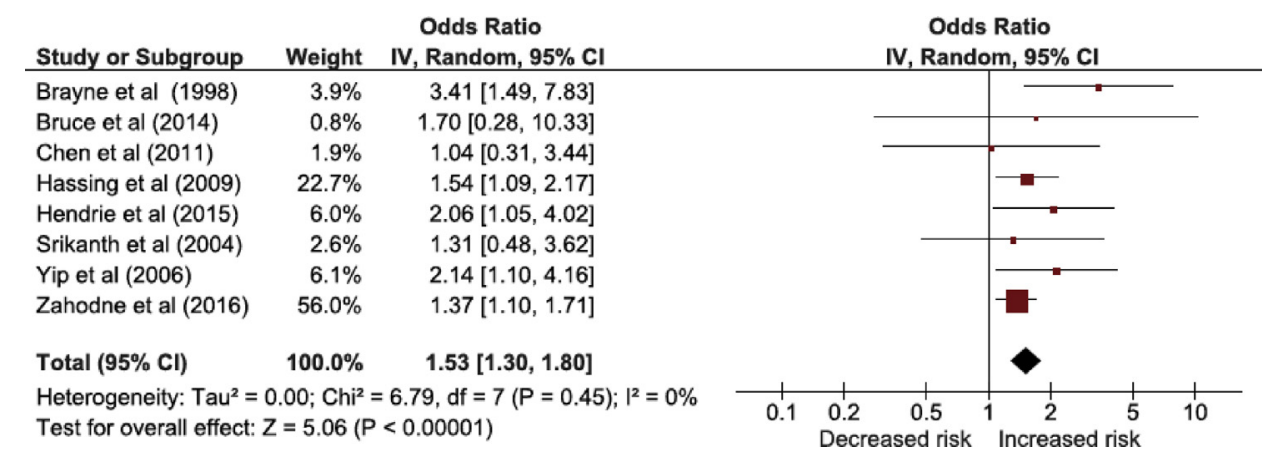

Fig. 3. Meta-analysis of odds ratios of prevalent stroke compared with no prevalent stroke on incident all-cause dementia. Data are presented as odds ratios with corresponding weight for each study in the meta-analysis because number of stroke events, dementia cases, and total number of participants were not always available in original included studies. The Supplementary Appendix shows the corresponding funnel plot. Abbreviations: CI, confidence interval; IV, inversevariance estimation method. 


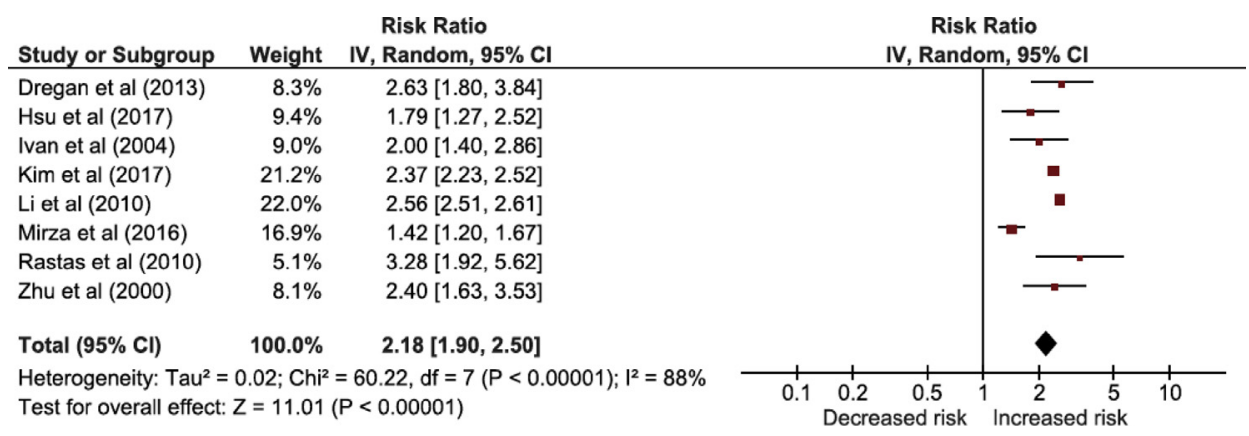

Fig. 4. Meta-analysis of risk ratios of incident stroke compared with no incident stroke on incident all-cause dementia. Data are presented as risk ratios with corresponding weight for each study in the meta-analysis because number of stroke events, dementia cases, and total number of participants were not always available in original included studies. The Supplementary Appendix shows the corresponding funnel plot. Abbreviations: CI, confidence interval; IV, inversevariance estimation method.

estimate $(\mathrm{RR}=2.14,95 \%$ CI: 0.64-7.13; Supplementary Appendix B, Table B3).

\subsection{Incident stroke}

Twelve prospective cohort studies [15,37,42,55-63] investigated the association between incident stroke and incident all-cause dementia (around 1.3 million participants and 131,217 stroke events; Supplementary Appendix B, Table B2). The majority of studies included older adults, and the analytic sample size ranged from 339 [62] to 799,069 [60]. One study [61] focused on the association with early onset dementia in men. In one additional study [60], 98\% of the participants were men.

When we combined adjusted results from eight studies $[15,37,55,57,59,60,62,63] \quad(849,059$ participants and 125,947 stroke events), the pooled estimate indicated that incident stroke more than doubled the risk of developing all-cause dementia compared with no incident stroke (pooled RR $=2.18,95 \%$ CI: $1.90-2.50, P<.001$, $\mathrm{I}^{2}=88 \%, 95 \%$ PI: 1.67-2.69; Fig. 4). No obvious sign of publication bias was detected by visual inspection of the funnel plot (Supplementary Appendix B, Fig. B4). None of the studies investigating incident stroke reported including participants with MCI at baseline. In a sensi- tivity analysis, we excluded three studies $[15,62,63]$ combining stroke with TIA. The pooled estimate was in the same direction though stronger, and the degree of heterogeneity between studies was slightly reduced (pooled RR $=2.41,95 \% \mathrm{CI}: 2.22-2.62, P<.001$, $\mathrm{I}^{2}=65 \%$, 95\% PI: 2.09-2.73; Supplementary Appendix B, Fig. B6.1). One study [56] reporting an adjusted OR could not be included in the meta-analyses, although their findings also suggested increased odds of incident dementia in those with incident stroke compared with no incident stroke (Supplementary Appendix B, Table B4). Meta-regression analyses indicated there was little evidence that inclusion of TIA in stroke assessment/diagnosis $(P=.49)$, mean/median age of participants $(P=.16)$, year at baseline examination $(P=.37)$, length of follow-up $(P=.32)$, or study quality $(P=.49)$ modified dementia risk.

In a secondary analysis, the pooled estimate for two studies [42,58] reporting unadjusted results (1007 participants and stroke events) indicated that incident stroke almost tripled the risk of dementia compared with no incident stroke (pooled RR $=2.96,95 \%$ CI: $1.81-4.84$, $P<.001, \mathrm{I}^{2}=33 \%$; Supplementary Appendix B, Fig. B6.2). A study focusing on early onset dementia in men [61] indicated that incident stroke almost tripled the

Table 2

Results for the effect of stroke and $A P O E \varepsilon 4$ on incident all-cause dementia compared with population without stroke and $A P O E \varepsilon 4$

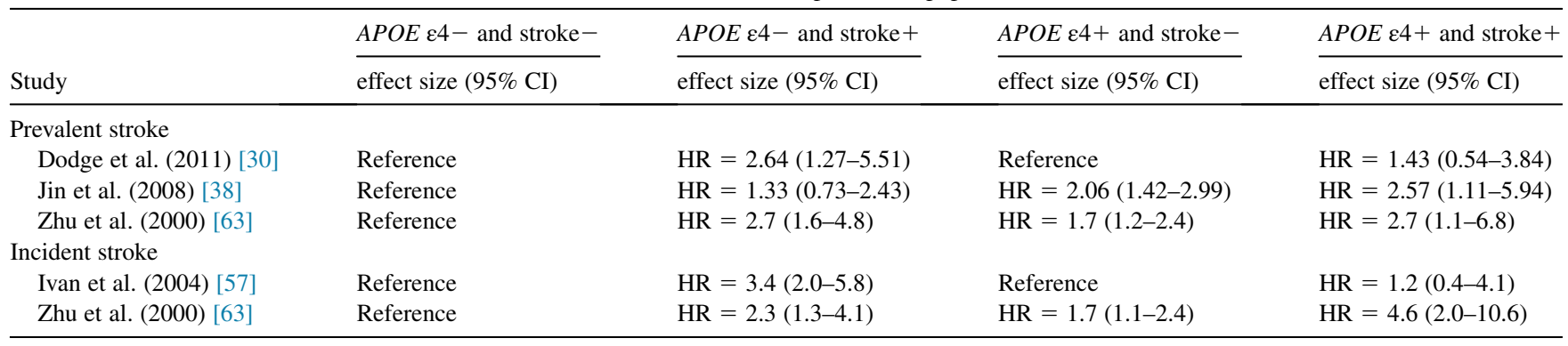

Abbreviations: $A P O E$, apolipoprotein E; CI, confidence interval; HR, hazard ratio. 
risk of developing early onset dementia (HR $=2.96,95 \%$ CI: 2.02-4.35; Supplementary Appendix B, Table B4).

\subsection{APOE genotype}

Three studies $[30,38,63]$ reported the combined effect of prevalent stroke and $A P O E \& 4$ on all-cause dementia risk for combinations of stroke and $A P O E$ genotype (Table 2). Prevalent stroke was associated with a significantly increased risk of dementia for $A P O E \varepsilon 4$ noncarriers in two out of three studies [30,63], and the HR for the nonsignificant association was in the same direction [38]. Similarly, two out of three studies of prevalent stroke in $A P O E$ \& 4 carriers indicated a significantly increased risk of dementia [38,63], and the HR of the nonsignificant association was again in the same direction [30]. However, there was no consistent difference in the effect sizes observed between $A P O E$ \& 4 carriers and noncarriers for prevalent stroke.

Two studies $[57,63]$ reported the combined effect of incident stroke and $A P O E \& 4$ on all-cause dementia risk for combinations of stroke and APOE genotype (Table 2). Incident stroke was associated with a significantly increased risk of dementia for $A P O E \& 4$ noncarriers in both the studies. One out of two studies found that incident stroke was associated with a significantly increased risk of dementia for $A P O E \& 4$ carriers [63], though the HR for the other study was in the same direction [57]. There was no consistent difference in the effect sizes observed between APOE $\varepsilon 4$ carriers and noncarriers for incident stroke.

\subsection{Sex-stratified findings}

Three studies $[25,43,57]$ reported additional results for incident all-cause dementia stratified by sex (Supplementary Appendix B, Table B6). One large cohort study [25] suggested a stronger association in men, whereas two further studies $[43,57]$ did not support a sex difference in the effect size.

\section{Discussion}

The results of our meta-analyses show that both prevalent and incident strokes are strong independent risk factors for all-cause dementia. However, significant betweenstudy heterogeneity was observed. Associations persisted when excluding studies that included participants with prevalent MCI or combined diagnosis of stroke with TIA. Stratified analyses did not suggest a consistent difference in the effect sizes observed between APOE $\varepsilon 4$ carriers and noncarriers for prevalent or incident stroke. Meta-regression analyses suggested that heterogeneity was not explained by a range of demographic factors or study characteristics, with the exception of sex which explained around half of the between-study variance observed for prevalent stroke.
Our meta-analyses extend the findings of the previous systematic review by Savva et al. [3] who concluded that stroke approximately doubles the risk of incident dementia in older adults. We included a larger number of prospective studies published since then (46 vs. 17), yielding a sample of nearly 3 million older adults, and we were able to provide pooled estimates for both prevalent and incident strokes in relation to the risk of all-cause dementia. Our results are also in line with a recent meta-analysis [5] of six studies reporting that participants with a history of stroke had 59\% increased risk of developing AD compared with controls. However, the aforementioned study did not include all-cause dementia as an outcome. Associations with increased rates of poststroke dementia are well known and have been previously synthesized [4]; our analysis extends these findings beyond poststroke incidence rates by providing pooled estimates for the risk of developing dementia compared to stroke-free populations.

Significant associations between stroke and higher risk of incident dementia were observed even after included studies adjusted for common modifiable risk factors for stroke such as hypertension, diabetes, myocardial infarction, and heart disease. Current evidence on the excess risk of stroke is based on observational data, and because it is not possible to randomize participants to stroke events, randomized controlled trials have only indirectly examined the effect of stroke prevention interventions on dementia risk reduction. For example, trials assessing the effect of antihypertensive therapy have reported reduced incidence of all-cause dementia, vascular dementia, and AD, but results are inconsistent [64,65]. Similarly, prospective studies on anticoagulation for secondary prevention of stroke in older adults with atrial fibrillation have shown variable effects on dementia risk [66,67]. Certain characteristics of stroke may explain the increased risk of dementia in stroke survivors. Studies investigating stroke subtypes have implicated both lacunar and hemorrhagic strokes as predictors of poststroke dementia $[4,68]$, but evidence is mixed, and variation in stroke subtyping methods may explain conflicting findings in the literature. The presence of multiple lesions, the volume of infarcts, and the location of stroke (e.g., left hemisphere) have also been identified as risk factors for poststroke dementia [4]. Neuroimaging studies have highlighted the role of medial temporal lobe atrophy and leukoaraiosis; extensive white matter changes related to subcortical stroke injury may increase the risk of memory decline and contribute to cortical gray matter thinning thereby increasing the risk of cognitive impairment [69]. Moreover, it has been suggested that stroke may trigger a neurodegenerative process by disrupting amyloid clearance [70] or by activating autoimmune responses [71] to brain antigens produced after stroke. It is also possible that existing AD pathology may predispose to stroke; neuroinflammation 
and compromised integrity of arterial walls related to accumulation of amyloid may result in greater risk of cerebrovascular events and increased infarct size [72]. It is therefore plausible that ongoing cerebrovascular injury due to vascular risk factors, immune processes, and pathogenic mechanisms may contribute to dementia risk after stroke.

This is the first meta-analysis to investigate the association of prevalent and incident strokes with incident allcause dementia. The strengths of this study include the comprehensive search strategy including major electronic databases, backward and forward citation searching, and contacting authors for relevant data. We included publications in which stroke was not the main variable of interest, and we were able to identify studies reporting nonsignificant results to counteract potential publication bias. We also performed meta-regression analyses to explore potential moderators that may explain between-study heterogeneity. We provide up-to-date evidence supporting associations between stroke and increased risk of dementia based on a large number of studies with long follow-up periods and millions of participants.

However, the present results should be considered in light of the limitations of the included original studies. Some studies included selective samples, for example, only men or women, volunteers, spouses of participants with stroke, and subsamples enrolled in specific projects. Although most studies reported dementia-free participants at baseline, we cannot exclude the possibility that more studies than those already identified in our analysis included populations with MCI and cognitive impairment. These biases may have led to an overestimation of the association between stroke and all-cause dementia. Nonetheless, current results were robust to sensitivity analysis when we excluded studies with known MCI cohorts (i.e., highly similar effect-size estimates). In addition, not all studies were specifically designed to investigate the association between prevalent or incident stroke and dementia. This translates into methodological differences in sample selection, stroke assessment and dementia diagnosis criteria, length of follow-up, statistical analysis plans, and adjustments to account for potential confounders. We were not able to incorporate important potential modifiers such as ethnicity and education in our meta-regression analyses due to inconsistent and incomplete reporting in the original studies. Clear and comprehensive reporting of information related to ethnic breakdown and educational level will facilitate harmonization of these potential modifiers across studies and subsequently strengthen future metaregression analyses. Only three studies used neuroimaging to define stroke status, and it is possible that techniques such as T2-weighted and fluid-attenuated inversion recovery magnetic resonance imaging and ${ }^{18} \mathrm{~F}$-2-fluoro-2-deoxy-D-glucose positron emission tomography [73] may help to reduce unexplained between-study variability by improving the quantification of stroke-related pathology, which in turn increases dementia risk. Similarly, unassessed variance in participant characteristics and the incidence of dementia unrelated to stroke may also have contributed to betweenstudy variability.

Finally, dementia may develop many years before the diagnosis, and in research studies, diagnosis is usually made during assessments at discrete times. Therefore, it is difficult to determine the exact period of dementia onset and as such the temporality of the association in studies of incident stroke and dementia especially in those with a long duration of follow-up. However, the stronger association observed for incident stroke suggests risk is greater near the time of stroke occurrence. More detailed reporting of the interval between stroke occurrence and dementia diagnosis in future studies will help to better characterize the role of time since stroke in the risk of dementia.

In conclusion, this systematic review and meta-analysis provides evidence that stroke is a strong independent risk factor for dementia. Given the consequences for people with dementia and their families and the significant implications for social and health-care costs, stroke prevention strategies should be integrated in multimodal health interventions to reduce dementia risk.

\section{Acknowledgments}

The authors thank all the authors of included original studies who provided additional data and clarifications for our analysis.

Funding: Authors are supported by the Mary Kinross Charitable Trust (D.J.L. and E.K.), the Halpin Trust (D.J.L., E.K., and I.L.), and the National Institute for Health Research (NIHR) Collaboration for Leadership in Applied Health Research and Care for the South West Peninsula (PenCLAHRC) (D.J.L., I.L., and O.C.U.). S.F.M. is supported by an NIHR-approved locally funded academic clinical fellowship. D.A.L. received grant support from National Institute on Aging (NIA)/National Institutes of Health (NIH) (R01 AG051827), and National Institute of Neurological Disorders and Stroke (NIH/NINDS; R01 NS102715). D.J.L. is supported by the NIA/NIH under award number RF1AG055654. The views expressed are those of the authors and not necessarily those of the Mary Kinross Charitable Trust, the Halpin Trust, the National Health Service, the NIHR, the Department of Health and Social Care, or the NIA/NIH.

Contributors: D.J.L. conceived and oversaw the study. E.K. and I.L. did the literature searches, reviewed all titles and abstracts, selected eligible studies, contacted authors, extracted data, planned and performed analyses, and cowrote the manuscript. S.F.M. assisted with data extraction, D.A.L. contributed substantial edits, and O.C.U. contributed to meta-analysis and meta-regression. All authors critically reviewed the final version of the manuscript. 


\section{Supplementary data}

Supplementary data related to this article can be found at https://doi.org/10.1016/j.jalz.2018.06.3061.

\section{RESEARCH IN CONTEXT}

1. Systematic review: To identify studies investigating the association between stroke and incident allcause dementia, we searched Medline, PsycINFO, and Embase; conducted backward and forward citation; contacted authors for additional data; and combined our findings with those from a 2008 systematic review.

2. Interpretation: Based on data describing more than 370,000 stroke events and over 3 million participants, we found robust evidence to support a significantly increased risk of all-cause dementia in those with a history of stroke. Even stronger associations were also evident for incident stroke, suggesting that it more than doubles the risk of all-cause dementia. Significant heterogeneity was observed, and the association with prevalent stroke appeared stronger in men.

3. Future directions: Our findings highlight the importance of stroke as an independent potentially modifiable risk factor for dementia. These findings were not accounted for by other vascular risk factors, and the stronger association for incident stroke suggests time since stroke may be important. Stroke characteristics and potential effect modifiers such as education and ethnicity warrant further investigation.

\section{References}

[1] Makin SDJ, Turpin S, Dennis MS, Wardlaw JM. Cognitive impairment after lacunar stroke: Systematic review and meta-analysis of incidence, prevalence and comparison with other stroke subtypes. J Neurol Neurosurg Psychiatr 2013;84:893-900.

[2] Pinkston JB, Alekseeva N, González Toledo E. Stroke and dementia. Neurol Res 2009;31:824-31.

[3] Savva GM, Stephan BC. Epidemiological studies of the effect of stroke on incident dementia: A systematic review. Stroke 2010; 41:e41-6.

[4] Pendlebury ST, Rothwell PM. Prevalence, incidence, and factors associated with pre-stroke and post-stroke dementia: A systematic review and meta-analysis. Lancet Neurol 2009;8:1006-18.

[5] Zhou J, Yu J-T, Wang H-F, Meng X-F, Tan C-C, Wang J, et al. Association between stroke and Alzheimer's disease: Systematic review and meta-analysis. J Alzheimers Dis 2015;43:479-89.

[6] Bellou V, Belbasis L, Tzoulaki I, Middleton LT, Ioannidis JP, Evangelou E. Systematic evaluation of the associations between envi- ronmental risk factors and dementia: An umbrella review of systematic reviews and meta-analyses. Alzheimers Dement 2017;13:406-18.

[7] Centre for Reviews and Dissemination (CRD). Systematic Reviews: CRD's Guidance for Undertaking Reviews in Health Care. Centre for Reviews and Dissemination 2009. York, UK: University of York; 2009.

[8] Thomas BH, Ciliska D, Dobbins M, Micucci S. A process for systematically reviewing the literature: Providing the research evidence for public health nursing interventions. Worldviews Evid Based Nurs 2004;1:176-84.

[9] Deeks JJ, Higgins JPT, Altman DG. Chapter 9: Analysing data and undertaking meta-analyses. In: Higgins JPT, Green S, eds. Cochrane Handbook for Systematic Reviews of Interventions. Chichester (UK): John Wiley \& Sons; 2008.

[10] Cochrane T. Review Manager (RevMan) 5.3 2008. Copenhagen: The Nordic Cochrane Centre; 2008.

[11] Higgins JP, Green S. Cochrane Handbook for Systematic Reviews of Interventions 2011. John Wiley \& Sons; 2011.

[12] Higgins JPT, Thompson SG, Deeks JJ, Altman DG. Measuring inconsistency in meta-analyses. BMJ 2003;327:557-60.

[13] Riley RD, Higgins JP, Deeks JJ. Interpretation of random effects metaanalyses. BMJ 2011;342:d549.

[14] Reitz C, Bos MJ, Hofman A, Koudstaal PJ, Breteler MM. Prestroke cognitive performance, incident stroke, and risk of dementia: the Rotterdam Study. Stroke 2008;39:36-41.

[15] Mirza SS, Portegies ML, Wolters FJ, Hofman A, Koudstaal PJ, Tiemeier H, et al. Higher education is associated with a lower risk of dementia after a Stroke or TIA. The Rotterdam Study. Neuroepidemiology 2016;46:120-7.

[16] American Psychiatric Association. Diagnostic and Statistical Manual of Mental Health Disorders (DSM-III-R) 1987. Washington, DC: American Psychiatric Association; 1987.

[17] American Psychiatric Association. In: Diagnostic and Statistical Manual. 4th ed. Washington, DC: American Psychiatric Association; 2000. Text Revision (DSM-IV-TR).

[18] World Health Organization. The ICD-10 Classification of Mental and Behavioural Disorders: Clinical Descriptions and Diagnostic Guidelines 1992. World Health Organization; 1992.

[19] Aguilar-Navarro SG, Mimenza-Alvarado AJ, Avila-Funes JA, JuarezCedillo T, Bernal-Lopez C, Hernandez-Favela CG. Clinical and demographic predictors of conversion to dementia in Mexican elderly with mild cognitive impairment. Rev Invest Clin 2017;69:33-9.

[20] Barnes DE, Beiser AS, Lee A, Langa KM, Koyama A, Preis SR, et al. Development and validation of a brief dementia screening indicator for primary care. Alzheimers Dement 2014;10:656-665.e1.

[21] Brayne C, Gill C, Huppert FA, Barkley C, Gehlhaar E, Girling DM, et al. Vascular risks and incident dementia: Results from a cohort study of the very old. Dement Geriatr Cogn Disord 1998;9:175-80.

[22] Bruce DG, Davis WA, Starkstein SE, Davis TM. Mid-life predictors of cognitive impairment and dementia in type 2 diabetes mellitus: The Fremantle Diabetes Study. J Alzheimers Dis 2014;3:S63-70.

[23] Chen R, Hu Z, Wei L, Ma Y, Liu Z, Copeland JR. Incident Dementia in a Defined Older Chinese Population. PLoS One 2011;6:e24817.

[24] Clerici F, Caracciolo B, Cova I, Imperatori SF, Maggiore L, Galimberti D, et al. Does vascular burden contribute to the progression of mild cognitive impairment to dementia? Dement Geriatr Cogn Disord 2012;34:235-43.

[25] Corraini P, Henderson VW, Ording AG, Pedersen L, Horvath-Puho E, Sorensen HT. Long-Term Risk of Dementia Among Survivors of Ischemic or Hemorrhagic Stroke. Stroke 2017;48:180-6.

[26] Crooks VC, Lubben J, Petitti DB, Little D, Chiu V. Social Network, Cognitive Function, and Dementia Incidence among elderly women. Am J Public Health 2008;98:1221-7.

[27] de Bruijn RF, Bos MJ, Portegies ML, Hofman A, Franco OH, Koudstaal PL, et al. The potential for prevention of dementia across two decades: The prospective, population-based Rotterdam Study. BMC Med 2015;13:132. 
[28] DeCarli C, Mungas D, Harvey D, Reed B, Weiner M, Chui H, et al. Memory impairment, but not cerebrovascular disease, predicts progression of MCI to dementia. Neurology 2004;63:220-7.

[29] Desmond DW, Moroney JT, Sano M, Stern Y. Incidence of dementia after ischemic stroke: Results of a longitudinal study. Stroke 2002; 33:2254-60.

[30] Dodge HH, Chang CC, Kamboh IM, Ganguli M. Risk of Alzheimer's disease incidence attributable to vascular disease in the population. Alzheimers Dement 2011;7:356-60.

[31] Downer B, Kumar A, Veeranki SP, Mehta HB, Raji M, Markides KS. Mexican-American dementia nomogram: Development of a dementia risk index for Mexican-American older adults. J Am Geriatr Soc 2016; 64:e265-9.

[32] Ganguli M, Lee C-W, Snitz BE, Hughes TF, McDade E, Chang C-CH. Rates and risk factors for progression to incident dementia vary by age in a population cohort. Neurology 2015;84:72-80.

[33] Hassing LB, Dahl AK, Thorvaldsson V, Berg S, Gatz M, Redersen NL, et al. Overweight in midlife and risk of dementia: A 40-year follow-up study. Int J Obes (2005) 2009;33:893-8.

[34] Hayden KM, Zandi PP, Lyketsos CG, Khachaturian AS, Bastian LA, Charoonruk G, et al. Vascular risk factors for incident Alzheimer disease and vascular dementia: The Cache County study. Alzheimer Dis Assoc Disord 2006;20:93-100.

[35] Hendrie HC, Hake A, Lane K, Purnell C, Unverzagt F, SmithGamble V, et al. Statin Use, Incident Dementia and Alzheimer Disease in Elderly African Americans. Ethn Dis 2015;25:345-54.

[36] Hobson P, Meara J. Cognitive function and mortality in a community-based elderly cohort of first-ever stroke survivors and control subjects. J Stroke Cerebrovasc Dis 2010;19:382-7.

[37] Hsu PF, Pan WH, Yip BS, Chen RC, Cheng HM, Chuang SY. C-reactive protein predicts incidence of dementia in an elderly Asian community cohort. JAMDA 2017;18:277.e7-277.e11.

[38] Jin YP, Ostbye T, Feightner JW, Di Legge S, Hachinski V. Joint effect of stroke and APOE 4 on dementia risk: the Canadian Study of Health and Aging. Neurology 2008;70:9-16.

[39] Kokmen E, Whisnant JP, O'Fallon WM, Chu CP, Beard CM. Dementia after ischemic stroke: A population-based study in Rochester, Minnesota (1960-1984). Neurology 1996;46:154-9.

[40] Kuller LH, Lopez OL, Newman A, Beauchamp NJ, Burke G, Dulberg C, et al. Risk factors for dementia in the cardiovascular health cognition study. Neuroepidemiology 2003;22:13-22.

[41] Li G, Shen YC, Chen CH, Zhau YW, Li SR, Lu M. A three-year follow-up study of age-related dementia in an urban area of Beijing. Acta Psychiatr Scand 1991;83:99-104.

[42] Liebetrau M, Steen B, Skoog I. Stroke in 85-year-olds: Prevalence, incidence, risk factors, and relation to mortality and dementia. Stroke 2003;34:2617-22.

[43] Noale M, Limongi F, Zambon S, Crepaldi G, Maggi S. Incidence of dementia: Evidence for an effect modification by gender. The ILSA Study. Int Psychogeriatr 2013;25:1867-76.

[44] Qiu C, Xu W, Winblad B, Fratiglioni L. Vascular risk profiles for dementia and Alzheimer's disease in very old people: A populationbased longitudinal study. J Alzheimers Dis 2010;20:293-300.

[45] Simons LA, Simons J, McCallum J, Friedlander Y. Lifestyle factors and risk of dementia: Dubbo Study of the elderly. Med J Aust 2006; 184:68-70.

[46] Srikanth VK, Anderson JF, Donnan GA, Saling MM, Didus E, Alpitsis R, et al. Progressive dementia after first-ever stroke: A community-based follow-up study. Neurology 2004;63:785-92.

[47] Srikanth VK, Quinn SJ, Donnan GA, Saling MM, Thrift AG. Long-term cognitive transitions, rates of cognitive change, and predictors of incident dementia in a population-based first-ever stroke cohort. Stroke 2006;37:2479-83.

[48] Tsai H-H, Yen R-F, Lin C-L, Kao C-H. Increased risk of dementia in patients hospitalized with acute kidney injury: A nationwide population-based cohort study. PLoS One 2017;12:e0171671.
[49] Walters K, Hardoon S, Petersen I, Illife S, Omar RZ, Nazareth I, et al. Predicting dementia risk in primary care: Development and validation of the Dementia Risk Score using routinely collected data. BMC Med 2016;14:6.

[50] Yamada M, Mimori Y, Kasagi F, Miyachi T, Ohshita T, Sasaki H. Incidence and risks of dementia in Japanese women: Radiation Effects Research Foundation Adult Health Study. J Neurol Sci 2009;283:57-61.

[51] Yip AG, Brayne C, Matthews FE. Risk factors for incident dementia in England and Wales: The Medical Research Council Cognitive Function and Ageing Study. A population-based nested case-control study. Age Ageing 2006;35:154-60.

[52] Zahodne LB, Schupf N, Brickman AM, Mayeux R, Wall MM, Stern Y, et al. Dementia risk and protective factors differ in the context of memory trajectory groups. J Attend Disord 2016;52:1013-20.

[53] Peters R, Poulter R, Beckett N, Forette F, Fagard R, Potter J, et al. Cardiovascular and biochemical risk factors for incident dementia in the Hypertension in the Very Elderly Trial. J Hypertens 2009;27:2055-62.

[54] Unverzagt FW, Guey LT, Jones RN, Marsiske M, King JW, Wadley VG, et al. ACTIVE cognitive training and rates of incident dementia. J Int Neuropsychol Soc 2012;18:669-77.

[55] Dregan A, Wolfe CD, Gulliford MC. Does the influence of stroke on dementia vary by different levels of prestroke cognitive functioning?: A cohort study. Stroke 2013;44:3445-51.

[56] Gamaldo A, Moghekar A, Kilada S, Resnick SM, Zonderman AB, O'Brien R. Effect of a clinical stroke on the risk of dementia in a prospective cohort. Neurology 2006;67:1363-9.

[57] Ivan CS, Seshadri S, Beiser A, Au R, Kase CS, Kelly-Hayes M, et al. Dementia after stroke: The Framingham Study. Stroke 2004;35:1264-8.

[58] Jin YP, Di Legge S, Ostbye T, Feightner JW, Hachinski V. The reciprocal risks of stroke and cognitive impairment in an elderly population. Alzheimers Dement 2006;2:171-8.

[59] Kim J-H, Lee Y. Dementia and death after stroke in older adults during a 10-year follow-up: Results from a competing risk model. J Nutr Health Aging 2018;22:297-301.

[60] Li N-C, Lee A, Whitmer RA, Kivipelto M, Lawler E, Kazis LE, et al. Use of angiotensin receptor blockers and risk of dementia in a predominantly male population: Prospective cohort analysis. BMJ 2010;340.

[61] Nordstrom P, Nordstrom A, Eriksson M, Wahlund LO, Gustafson Y. Risk factors in late adolescence for young-onset dementia in men: A nationwide cohort study. JAMA Intern Med 2013;173:1612-8.

[62] Rastas S, Pirttila T, Mattila K, Verkkoniemi A, Juva K, Niinisto L, et al. Vascular risk factors and dementia in the general population aged $>85$ years: Prospective population-based study. Neurobiol Aging 2010; $31: 1-7$.

[63] Zhu L, Fratiglioni L, Guo Z, Basun H, Corder EH, Winblad B, et al. Incidence of dementia in relation to stroke and the apolipoprotein E epsilon4 allele in the very old. Findings from a population-based longitudinal study. Stroke 2000;31:53-60.

[64] Rouch L, Cestac P, Hanon O, Cool C, Helmer C, Bouhanick B, et al. Antihypertensive drugs, prevention of cognitive decline and dementia: a systematic review of observational studies, randomized controlled trials and meta-analyses, with discussion of potential mechanisms. CNS Drugs 2015;29:113-30.

[65] Román GC. Vascular dementia prevention: A risk factor analysis. Cerebrovasc Dis 2005;2:91-100.

[66] Barber M, Tait R, Scott J, Rumley A, Lowe G, Stott D. Dementia in subjects with atrial fibrillation: Hemostatic function and the role of anticoagulation. J Thromb Haemost 2004;2:1873-8.

[67] Bunch TJ, May HT, Bair TL, Crandall BG, Cutler MJ, Day JD, et al. Atrial fibrillation patients treated with long-term warfarin anticoagulation have higher rates of all dementia types compared with patients receiving long-term Warfarin for other indications. J Am Heart Assoc 2016;5(7):e003932.

[68] Béjot Y, Aboa-Eboulé C, Durier J, Rouaud O, Jacquin A, Ponavoy E, et al. Prevalence of early dementia after first-ever stroke. Stroke 2011; 42:607-12. 
[69] Kalaria RN, Akinyemi R, Ihara M. Stroke injury, cognitive impairment and vascular dementia. Biochim Biophys Acta 2016;1862:915-25.

[70] Garcia-Alloza M, Gregory J, Kuchibhotla KV, Fine S, Wei Y, Ayata C, et al. Cerebrovascular lesions induce transient $\beta$-amyloid deposition. Brain 2011;134:3697-707.

[71] Doyle KP, Buckwalter MS. Does B lymphocyte-mediated autoimmunity contribute to post-stroke dementia? Brain Behav Immun 2016;64:1-8.
[72] Whitehead SN, Cheng G, Hachinski VC, Cechetto DF. Progressive increase in infarct size, neuroinflammation, and cognitive deficits in the presence of high levels of amyloid. Stroke 2007; 38:3245-50.

[73] Heiss WD, Rosenberg GA, Thiel A, de Reuck J. Neuroimaging in vascular cognitive impairment: A state-of-the-art review. BMC Med 2016;14:174.

\section{Did you know?}

You can track the impact of your article with citation alerts that let you know when your article (or any article you'd like to track) has been cited by another Elsevier-published journal. 